\title{
Error-tolerant Resource Allocation and Payment Minimization for Cloud System
}

\author{
Sheng Di, Member, IEEE, and Cho-Li Wang, Member, IEEE
}

\begin{abstract}
With virtual machine (VM) technology being increasingly mature, compute resources in Cloud systems can be partitioned in fine granularity and allocated on demand. We make three contributions in this paper: (1) We formulate a deadline-driven resource allocation problem based on the Cloud environment facilitated with VM resource isolation technology, and also propose a novel solution with polynomial time, which could minimize users' payment in terms of their expected deadlines. (2) By analyzing the upper bound of task execution length based on the possibly inaccurate workload prediction, we further propose an error-tolerant method to guarantee task's completion within its deadline. (3) We validate its effectiveness over a real VM-facilitated cluster environment under different levels of competition. In our experiment, by tuning algorithmic input deadline based on our derived bound, task execution length can always be limited within its deadline in the sufficient-supply situation; the mean execution length still keeps $70 \%$ as high as user-specified deadline under the severe competition. Under the original-deadline-based solution, about $52.5 \%$ of tasks are completed within $0.95 \sim 1.0$ as high as their deadlines, which still conforms to the deadline-guaranteed requirement. Only $20 \%$ of tasks violate deadlines, yet most (17.5\%) are still finished within 1.05 times of deadlines.
\end{abstract}

Keywords-VM-multiplexing, Resource Allocation, Convex Optimization, Prediction Error Tolerance, Payment Minimization

\section{INTRODUCTION}

Cloud computing [1], [2] has emerged as a compelling paradigm for the deployment of ease-of-use virtual environment on the Internet. One typical feature of Clouds is its pool of easily accessible virtualized resources (such as hardware, platform or services) that can be dynamically reconfigured to adjust to a variable load (scale). All the resources provisioned by Cloud system are supposed to be under a payment model [2], in order to avoid users' over-demand of their resources against their true needs.

Each task's workload is likely of multiple dimensions. First, the compute resources in need may be multiattribute (such as CPU, disk-reading speed, network bandwidth, etc.), resulting in multi-dimensional execution in nature. Second, even though a task just depends on one resource type like CPU, it may also be split to multiple sequential execution phases, each calling for a different computing ability and various price on demand, also leading to a potentially high-dimensional execution scenario.

The resource allocation in Cloud computing is much more complex than in other distributed systems like Grid computing platform. In a Grid system [3], it is improper to share the compute resources among the multiple applications simultaneously running atop it due to the inevitable mutual performance interference among them. Whereas, Cloud systems usually do not provision physical hosts directly to users, but leverage virtual resources isolated by VM technology [4], [5], [6].

- S. Di is currently a post-doctor researcher at INRIA, Grenoble, France, and C.L. Wang is with the Department of Computer Science, The University of Hong Kong, Hong Kong.
Not only can such an elastic resource usage way adapt to user's specific demand, but it can also maximize resource utilization in fine granularity and isolate the abnormal environments for safety purpose. Some successful platforms or cloud management tools leveraging VM resource isolation technology include Amazon EC2 [7] and OpenNebula [8]. On the other hand, with fast development of scientific research, users may propose quite complicated demands. For example, users may wish to minimize their payments when guaranteeing their service level such that their tasks can be finished before deadlines. Such a deadline-guaranteed resource allocation with minimized payment is rarely studied in literatures. Moreover, inevitable errors in predicting task workloads will definitely make the problem harder.

Based on the elastic resource usage model, we aim to design a resource allocation algorithm with high prediction-error tolerance ability, also minimizing users' payments subject to their expected deadlines.

Since the idle physical resources can be arbitrarily partitioned and allocated to new tasks, the VM-based divisible resource allocation could be very flexible. This implies the feasibility of finding the optimal solution through convex optimization strategies [9], unlike the traditional Grid model [10] that relies on the indivisible resources like the number of physical cores. However, we found it is inviable to directly solve the necessary and sufficient condition to find the optimal solution, a.k.a., Karush-Kuhn-Tucker (KKT) conditions [9]. Our first contribution is devising a novel approach (with only $O\left(n \cdot R^{2}\right)$ time complexity) to solve the problem, where $R$ denotes the number of execution dimensions and $n$ is the system scale (the number of compute nodes).

In literatures, traditional optimization problems are 
often subject to the precise prediction of task's characteristic (or execution property), which is nontrivial to realize in practice. Accordingly, as the state-of-the-art, we further analyze our algorithm's optimality approximation ratio given the possibly wrong predictions of tasks' execution properties. In particular, we will try to answer such a question: when application's characteristic is predicted with certain levels of errors, will the application's final execution length (a.k.a., execution time) violate (or surpass) its deadline? If yes, what is the ratio of the final execution time to its deadline? These theoretical results will be significantly valuable to the guarantee of user's service level in practice. In fact, by setting a relatively stricter deadline properly based on our derived approximation ratio, each task can be guaranteed to be finished within its original deadline even though task properties cannot be predicted accurately.

In addition to the above theoretical contribution, we further confirm the effectiveness of our solutions by implementing a set of advanced web services that are based on complex matrix-operations, over a real cluster environment with 60 virtual machines. All the theoretical conclusions are confirmed with our experiments. Specifically, in the situation with relatively sufficient resources, the worst-case tasks under the stricter-deadline based allocation only take as about 0.75 times as their deadlines to complete, as compared to the 1.2 times of the deadlines under the original user-predefined deadline based allocation. We also observe that in the competitive environment, the latter algorithm performs much more stable than the former instead, which means that the latter tolerates the resource competition better. We also confirm the effectiveness of our solution via the distribution of the number of tasks with respect to execution times and user payments: in the competitive situation, majority of tasks can be guaranteed to be completed within deadlines.

The rest of the paper is organized as follows: In Section 2, we formulate our problem based on the Cloud scenario which supports elastic divisible resource customization. In Section 3, we first discuss the complexity of the modeled problem in brief, and then formally describe a novel algorithm, which can minimize user's payment based on task's preset execution deadline. In Section 4, we intensively derive the lower bound and upper bound of execution time for the situation with the possibly skewed predictions on tasks' properties as compared to the deadlines. We rigorously implement our algorithm and analyze experimental results on a realcluster setting in Section 5. We discuss the related works in Section 6 and conclude with future work in Section 7.

\section{Problem Formulation}

In Cloud systems, the Cloud proxy (a.k.a., server) continually receives and responds to user requests (or tasks) with customized requirements (or virtual machines). All tasks will be handled based on their priorities (like Google task scheduler [11]) or in terms of First-ComeFirst-Serve (FCFS) policy when the tasks are of the same priorities (like [12]). Each task's execution may involve multi-dimensional resources, such as CPU and disk I/O. A data mining task, for example, usually needs to load a large set of data from disk before or in the middle of its computation. Eventually, such a task may store its computation results onto the local disk or a public server through network. Fig. 1 illustrates the procedure in processing such a task (denoted $t_{i}$ ). Suppose the task's execution times cost on computation and disk processing are predicted as 4 hours and 3 hours respectively. Upon receiving the request, the scheduler checks the pre-collected availability states of all candidate nodes, and estimates the minimal payment of running the task within its deadline on each of them (i.e., Step 1 in the figure). The host (Node $p_{3}$ shown in Fig. 1) that requires the lowest payment will run the task via a customized VM instance with isolated resources (Step 2 in Fig. 1). Specifically, the VM will be customized with such a CPU rate (e.g., 0.4 Gflops) and disk I/O rate (e.g., 0.3 Gbps) that the task can be finished within its deadline $\left(D\left(t_{i}\right)=1\right.$ hour in the example) and its user payment can also be minimized meanwhile. Finally (Step 3), its computation results (or feedbacks) will be returned to users.

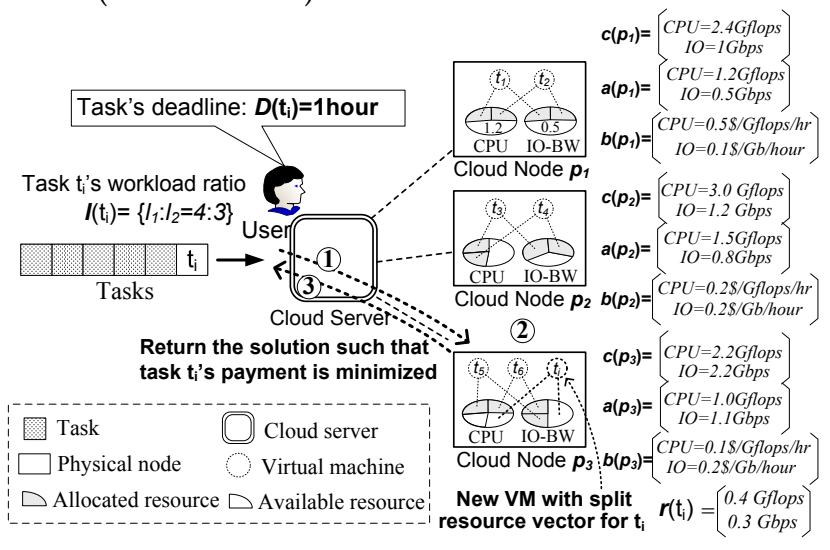

Fig. 1: Resource Allocation in Cloud System

Suppose there are $n$ compute nodes (denoted by $p_{i}$, where $1 \leq i \leq n)$. Since all the resources are managed centrally, the availability state of each resource within any recent or later period can be predicted prior, for executing any given task with multiple execution dimensions. For any particular task with $R$ execution dimensions, we use $\Pi$ to denote the whole set of dimensions and $\boldsymbol{c}\left(p_{i}\right)=\left(c_{1}\left(p_{i}\right), c_{2}\left(p_{i}\right), \cdots, c_{R}\left(p_{i}\right)\right)^{T}$ as node $p_{i}$ 's capacity vector on these dimensions (In the paper, we use bold-type to indicate a vector). In Fig. 1 , for example, node $p_{1}$ 's physical capacity vector is $c\left(p_{1}\right)=\{\mathrm{CPU}=2.4 \mathrm{Gflops}$,disk_IO=1Gbps .

Any user's task is denoted as $t_{i}$, where $1 \leq i \leq m$, and $m$ refers to the total number of submitted tasks. Each task has a multi-dimensional workload vector, denoted by $\boldsymbol{l}\left(t_{i}\right)=\left(l_{1}\left(t_{i}\right), l_{2}\left(t_{i}\right), \cdots, l_{R}\left(t_{i}\right)\right)^{T}$, which needs to be finished before the task's deadline. We denote the resource vector allocated to $t_{i}$ as $\boldsymbol{r}\left(t_{i}\right)=\left(r_{1}\left(t_{i}\right), r_{2}\left(t_{i}\right)\right.$, $\left.\cdots, r_{R}\left(t_{i}\right)\right)^{T}$, where $r_{k}\left(t_{i}\right)(k=1,2, \cdots, R)$ refers to the resource amount on $k$ th execution dimension isolated 
by hypervisor/virtual machine monitor(VMM) for the task's execution. Node $p_{i}$ 's availability vector (denoted $\boldsymbol{a}\left(p_{i}\right)$ ) along the multiple dimensions is calculated by $\boldsymbol{c}\left(p_{j}\right)-\sum_{t_{i} \text { running on } p_{j}} \boldsymbol{r}\left(t_{i}\right)$. For example, node $p_{1}$ in Fig. 1 is running two VMs that are allocated with half of the total physical resources, so its availability vector $\boldsymbol{a}\left(p_{1}\right)=\{\mathrm{CPU}=1.2 \mathrm{Gflops}$,disk_IO $=0.5 \mathrm{Gbps}\}$. If there are no workloads being executed simultaneously for a particular task, its total execution time will be the sum of the individual processing times on different dimensions. If the execution of the workloads overlap, however, the task's completion time would be shorter. Accordingly, $t_{i}$ 's final execution time (denoted as $T\left(t_{i}\right)$ ) is definitely confined within such a range $\left[\max \left(\frac{l_{k}}{r_{k}}\right), \sum_{k=1}^{R} \frac{l_{k}}{r_{k}}\right]$. For simplicity, we denote task $t_{i}$ 's execution time as Equation (1) (affine transformation of $\sum_{i=1}^{R} \frac{l_{k}}{r_{k}}$ ), where $\theta$ denotes a constant coefficient. Such a definition specifies a defacto broad set of applications each with multiple execution dimensions. The typical example is a single job with multiple sequentially interdependent tasks or some program with distinct execution phases each relying on independent compute resources (where $\theta=1$ ).

$$
T\left(t_{i}\right)=\theta \sum_{k=1}^{R} \frac{l_{k}\left(t_{i}\right)}{r_{k}\left(t_{i}\right)} \text {, where } \theta \in\left[\frac{\max \left(\frac{l_{k}}{r_{k}}\right)}{\sum_{k=1}^{R} \frac{l_{k}}{r_{k}}}, 1\right]
$$

For any Cloud system, the resources provisioned are usually set with a price vector denoted as $\boldsymbol{b}\left(p_{i}\right)=\left(b_{1}\left(p_{i}\right)\right.$, $\left.b_{2}\left(p_{i}\right), \cdots, b_{R}\left(p_{i}\right)\right)^{T}$ along $R$ dimensions. $b_{k}\left(p_{i}\right)(1 \leq k$ $\leq R$ ) denotes the per-time-unit price that the consumers need to pay for the consumption of the $k$ th dimension on $p_{i}$. Each task $t_{i}$ is set with a deadline (denoted $D\left(t_{i}\right)$ ) for its execution and the payment is expected to be minimized under our algorithm.

In our Cloud model, any task will be executed on one or more virtual machines with user-reserved resources and the payment is calculated based on the customized resource (a.k.a., pay-by-reserve policy). Adopting such a pricing policy is driven by three reasons. Firstly, the efficiencies of many applications usually rely on multiple resources but it is non-trivial to precisely evaluate the exact amount of their consumption separately on individual resources. Secondly, quite a few users prefer to reserving resources for tolerating usage burst and guaranteeing their service levels. Lastly, the alternative pricing policy, pay-as-you-consume, is rather simple because its payment is always fixed (= $\left.\theta \sum_{k=1}^{R}\left(b_{k}\left(p_{s}\right) \cdot r_{k}\left(t_{i}\right) \frac{l_{k}\left(t_{i}\right)}{r_{k}\left(t_{i}\right)}\right)=\theta \sum_{k=1}^{R} b_{k}\left(p_{s}\right) \cdot l_{k}\left(t_{i}\right)\right)$ regardless of the resource allocation.

Based on the pay-by-reserve policy, task $t_{i}$ 's total payment will be calculated via Equation (2), where $p_{s}$ refers to $t_{i}$ 's execution node. The mean price (i.e., $\left.\frac{1}{R} \boldsymbol{b}\left(p_{s}\right)^{T} \cdot \boldsymbol{r}\left(t_{i}\right)\right)$ will be used as the pricing unit over time, for computing user's payment. Such a design can be consistent with our pay-by-reserve model, and also prevent users from feeling too costly when their applications' execution cannot overlap at different dimensions.

$$
P\left(\boldsymbol{r}\left(t_{i}\right)\right)=\frac{1}{R} \boldsymbol{b}\left(p_{s}\right)^{T} \cdot \boldsymbol{r}\left(t_{i}\right) \cdot T\left(t_{i}\right)
$$

In this paper, we might omit the notations $t_{i}$ and $p_{i}$ if thus would not cause ambiguity. For instance, $l_{k}\left(t_{i}\right)$, $\boldsymbol{r}\left(t_{i}\right), b_{k}\left(p_{i}\right), \boldsymbol{a}\left(p_{i}\right)$ and $D\left(t_{i}\right)$ may be substituted by $l_{k}, \boldsymbol{r}$, $b_{k}, a$, and $D$ respectively, in the following text.

Our research could be briefly summarized as the following convex optimization format: for any task $t_{i}$ with its workload vector $\boldsymbol{l}\left(t_{i}\right)$, given a set of candidate execution nodes $\left(p_{s}, s=1,2, \cdots, n\right)$, how to select $p_{s}$ and split resources such that $t_{i}$ 's payment (i.e., Equation (2)) is minimized, subject to the constraints (3) and (4).

$$
\begin{aligned}
& \text { Min } P\left(\boldsymbol{r}\left(t_{i}\right)\right) \\
& \text { s.t. } \\
& T\left(t_{i}\right) \leq D\left(t_{i}\right) \\
& \boldsymbol{r}\left(t_{i}\right) \preceq \boldsymbol{a}\left(p_{s}\right)
\end{aligned}
$$

\section{Optimal Resource Allocation}

In this section, we will first analyze the problem mentioned above, and then propose our optimal solution.

By combining Equation (1) and Equation (2), it is easy to verify that $\forall r_{k}, \frac{\partial^{2} P\left(\boldsymbol{r}\left(t_{i}\right)\right)}{\partial r_{k}^{2}}=\frac{\theta}{R}\left(-\frac{2 b_{k} l_{k}}{r_{k}^{2}}+\frac{2 l_{k}}{r_{k}^{3}} \sum_{i=1}^{R} b_{i} r_{i}\right)>0$, thus the target function $P\left(\boldsymbol{r}\left(t_{i}\right)\right)$ is convex, which means that there must exist a minimal extreme point.

Based on the convex optimization theory [9], the Lagrangian function of the problem could be formulated as Equation (5), where $\lambda$ and $\mu_{1}, \mu_{2}, \cdots, \mu_{R}$ are corresponding Lagrangian multipliers. Note that $\theta$ is a constant defined in Equation (1) and $r$ is the abbreviation of $r\left(t_{i}\right)$ as stated above.

$F_{1}(\boldsymbol{r})=\frac{1}{R}\left(\sum_{k=1}^{R} b_{k} r_{k}\right)\left(\theta \sum_{k=1}^{R} \frac{l_{k}}{r_{k}}\right)+\lambda\left(\theta \sum_{k=1}^{R} \frac{l_{k}}{r_{k}}-D\right)+\sum_{k=1}^{R} \mu_{k}\left(r_{k}-a_{k}\right)$

Accordingly, we could get the Karush-Kuhn-Tucker (KKT) conditions [9] (i.e., the necessary and sufficient condition of the optimization) as below:

$$
\left\{\begin{array}{l}
\lambda \geq 0, \mu_{k} \geq 0, k=1,2 \cdots, R \\
\sum_{i=1}^{R} \theta \frac{l_{i}}{r_{i}} \leq D \\
\lambda\left(\theta \sum_{i=1}^{R} \frac{l_{i}}{r_{i}}-D\right)=0 \\
r_{k} \leq a_{k}\left(p_{s}\right), k=1,2, \cdots, R ; s=1,2, \cdots, n \\
\mu_{k}\left(r_{k}-a_{k}\left(p_{s}\right)\right)=0, k=1,2, \cdots, R ; s=1,2, \cdots, n \\
\frac{\partial F_{1}}{\partial r_{k}}=\frac{1}{R}\left(\left(\sum_{i=1}^{R} b_{i} r_{i}\right) \cdot \frac{-l_{k}}{r_{k}^{2}}+b_{k} \cdot \sum_{i=1}^{R} \frac{l_{i}}{r_{i}}+\frac{-\lambda l_{k}}{r_{k}^{2}}+\mu_{k}\right)=0 \\
\quad k=1,2, \cdots, R
\end{array}\right.
$$

In other words, as long as we can find such an allocation case $\left(r=\left(r_{1}, r_{2}, \cdots, r_{R}\right)^{T}\right)$ to satisfy the above conditions simultaneously, we can set it as the optimal solution of the deadline-driven payment-minimized problem. However, it is non-trivial to do that, because the last condition $\left(\frac{\partial F_{1}}{\partial r_{k}}=0\right)$ cannot be directly solved. Whereas, we exploit a novel algorithm with polynomial time complexity $\left(n \cdot R^{2}\right)$ to allocate resource, which can be proved to satisfy the KKT condition listed above.

Our algorithm is designed based on such a discovery: if we do not consider the limit of resource capacities (i.e condition (4)), the problem can be directly solved using Lagrangian multiplier method. As follows, we will 
first derive the optimal solution to the problem with unbounded capacities (i.e., without the condition (4)) in Theorem 1. And then, we will describe our algorithm by recursively using Theorem 1 to search the resource allocation case that satisfies the whole KKT condition (6), in polynomial time.

Theorem 1: For a specific task $t_{i}$, in order to minimize $P\left(r\left(t_{i}\right)\right)$ subject to the constraint (3), the optimal resource vector $\boldsymbol{r}^{(*)}\left(t_{i}\right)$ is Equation (7), where $k=1,2, \cdots, R$. (Note that $r^{(*)}\left(t_{i}\right)$ is not subject to Inequality (4), unlike the notation $\boldsymbol{r}^{*}\left(t_{i}\right)$ that takes into account this inequality.)

$$
r_{k}^{(*)}\left(t_{i}\right)=\left(\frac{\theta}{D} \sum_{j=1}^{R} \sqrt{l_{j} b_{j}}\right) \sqrt{\frac{l_{k}}{b_{k}}}
$$

Proof: As mentioned previously, the target function is convex, thus there must exist the minimal extreme point. In order to simplify the target function (i.e., Equation (2)), we fix the task's execution time to be $T(\leq D)$, which also satisfies the problem's conditions. Then, the target function could be converted to Equation (8).

$$
P(\boldsymbol{r})=\frac{T}{R} \cdot \sum_{k=1}^{R} b_{k} r_{k} \text {, where } T \leq D
$$

The corresponding Lagrangian function is shown below:

$$
F_{2}(\boldsymbol{r})=\frac{T}{R} \cdot \sum_{k=1}^{R} b_{k} r_{k}+\lambda\left(\theta \sum_{k=1}^{R} \frac{l_{k}}{r_{k}}-D\right)
$$

Based on the Lagrangian multiplier method, $\frac{\partial F_{2}}{\partial r_{k}}=0$ (where $k=1,2, \cdots, R$ ) constructs a set of necessary conditions for getting the optimal solution (i.e., Equation (10) must hold, where $\lambda$ is a constant).

$$
\lambda \theta R / T=b_{k} r_{k}^{2} / l_{k}
$$

According to Equation (10), we can easily get Equation (11), $\forall j, k(1 \leq j \neq k \leq R)$.

$$
r_{k}^{2} b_{k} / l_{k}=r_{j}^{2} b_{j} / l_{j}
$$

That is, Equation (12) is the sufficient and necessary condition of the optimal solution, s.t. a given deadline.

$$
r_{1}: r_{2}: \cdots r_{R}=\sqrt{l_{1} / b_{1}}: \sqrt{l_{2} / b_{2}}: \cdots: \sqrt{l_{R} / b_{R}}
$$

In order to save the resource utilized by the current task as much as possible, the optimal allocation should make $\sum_{i=1}^{R} \frac{l_{i}}{r_{i}}$ equal to $D$. In fact, for any resource allocation $\boldsymbol{r}\left(t_{i}\right)$ meeting Equation (12) while $\sum_{i=1}^{R} \frac{l_{i}}{r_{i}}<$ $D$, there must exist another solution with lower resource allocation $\boldsymbol{r}\left(t_{i}\right)^{\prime}$ (i.e., $\left.\boldsymbol{r}^{\prime}\left(t_{i}\right) \preceq \boldsymbol{r}\left(t_{i}\right)\right)$ such that it also satisfies Equation (12). Hence, the task $t_{i}$ 's optimal resource allocation should make $\sum_{k=1}^{R} \frac{l_{k}}{r_{k}}=D$, then, by combining this equation, we can calculate the the optimal resource vector to be allocated as Equation (7).

Remark: With unbounded resource availabilities, there will be no any constraint to the problem of minimizing the target function $P(\boldsymbol{r})$. Based on the above analysis, there are infinite number of optimal stationary points, whose sufficient and necessary conditions are Equation (12). For vivid illustration, we show the graph of a simple case in Fig. 2, where $\boldsymbol{b}=(1,1)^{T}$ and $\boldsymbol{l}=(1,1)^{T}$. From this figure, we can observe that these exist the minimal extreme points and the number of them is infinitive, along the line $\left\{r_{1}=r_{2}\right.$ and $\left.P(\boldsymbol{r})=4\right\}$. This result is consistent with the Equation (12).

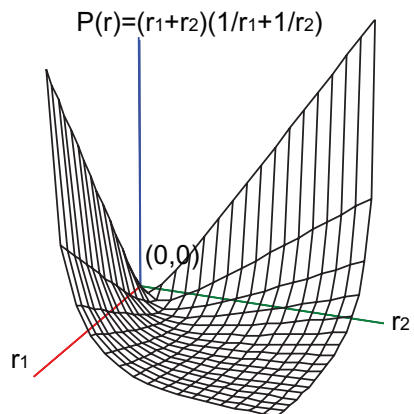

Fig. 2: The function Graph of A Simple Case

Formula (7) presents the resource share vector $r^{(*)}$ gained by $t_{i}$ such that its payment and the resource utilization can be both minimized within its execution deadline (i.e., Formula (3)). Considering the constraint (4), $\boldsymbol{r}^{(*)}$ is right the optimal solution as long as $\boldsymbol{r}^{(*)} \preceq \boldsymbol{a}\left(p_{s}\right)$. However, if $\boldsymbol{r}^{(*)}$ does not fully satisfy the constraint (4) (i.e., $\left.\exists k: r_{k}^{(*)}>a_{k}\left(p_{s}\right)\right), r^{(*)}$ should not be a feasible solution. As one contribution, we propose an efficient algorithm (Algorithm 1) to determine the optimal solution subject to the constraint (4) with the provable time complexity $O\left(n \cdot R^{2}\right)$.

Definition 1: For any task $t_{i}$, based on a subset $\Gamma(\subseteq \Pi)$, $\operatorname{Co-STEP}(\Gamma, C)$ is defined as the procedure of computing the optimal solution of minimizing $P\left(r_{\Gamma}\left(t_{i}\right)\right)$ subject to the constraint (13) by using convex optimization (similar to the proof of Theorem 1), where $C$ denotes a deadline and $\boldsymbol{r}_{\Gamma}\left(t_{i}\right)\left(=\left(r_{1}, r_{2}, \cdots, r_{R}\right)^{T}\right)$ denotes the resource shares gained by $t_{i}$ on the execution dimension set $\Gamma$.

$$
\theta \sum_{i=1}^{R} \frac{l_{i}}{r_{i}} \leq C
$$

We devise Algorithm 1 for minimizing $P\left(\boldsymbol{r}\left(t_{i}\right)\right)$ subject to the constraints (3) and (4), as shown below.

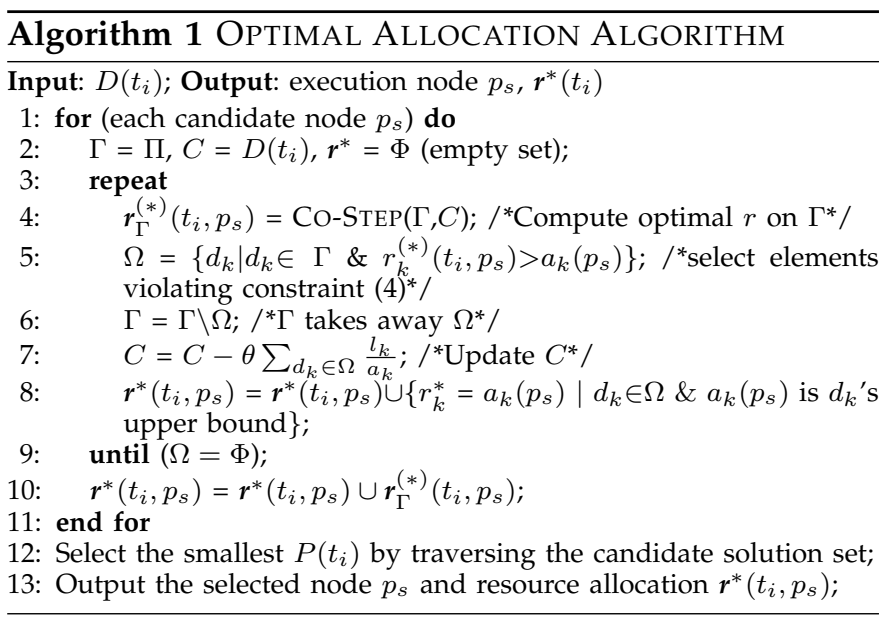

In this algorithm, line 4 executes $\operatorname{CO}-\operatorname{STEP}(\Gamma, C)$ in order to find the optimal $\boldsymbol{r}_{\Gamma}^{(*)}\left(t_{i}, p_{s}\right)$, under the assumption without constraint (4). If $r_{\Gamma}^{(*)}\left(t_{i}, p_{s}\right)$ completely satisfies the constraint (4) (i.e., $\Omega=\Phi)$, then $r_{\Gamma}^{(*)}\left(t_{i}, p_{s}\right)$ is the local optimal resource allocation for $t_{i}$ to be run on $p_{s}$; otherwise, let the resource shares $\left(r_{k}\left(t_{i}\right)\right.$,where $k=1,2, \cdots, R)$ that violate the constraint (4) equal to its 
upper bound (i.e., $a_{k}\left(p_{s}\right)$ ) and take the corresponding execution dimensions (i.e., $\Omega$ ) away from $\Gamma$, then, $C$ $=C-\theta \sum_{d_{k} \in \Omega} \frac{l_{k}}{a_{k}}$ for the remaining dimensions. The process will go on until the computed optimal resource shares on the remaining dimensions satisfy the constraint (4). Since the time complexity of $\operatorname{CO}-\operatorname{SteP}(\Gamma, C)$ is $O(|\Gamma|)$, the number of computation steps of line $2 \sim 10$ in Algorithm 1 in the worst case is $\sum_{i=0}^{R-1}(R-i)$, thus the total time complexity of Algorithm $1=O\left(n \cdot R^{2}\right)$.

Based on the Algorithm 1, it is obvious that the local optimal resource allocation for $t_{i}$ to be executed on a specified node $p_{s}$ is the most crucial part. In fact, the final outputted resource allocation solution of the whole algorithm will be globally optimal around the whole system as long as each local process on a specified node (line 2 10) can be proved as optimal resource allocation. Consequently, we will intensively discuss the local divisible-resource allocation by specifying a particular execution node, in the following text.

Theorem 2: Given a submitted task $t_{i}$ with its load vector $\boldsymbol{l}\left(t_{i}\right)$ and a deadline $D\left(t_{i}\right)$ and a particular node $p_{s}$ with its resource price vector $\boldsymbol{b}\left(p_{s}\right)$, then the output after running the line $2 \sim 10$ of Algorithm 1 (i.e., $\boldsymbol{r}^{*}\left(t_{i}, p_{s}\right)$ is optimal for minimizing $t_{i}$ 's payment (i.e., $P\left(r\left(t_{i}\right)\right)$ ), subject to the constraints (3) and (4).

Main idea: We will prove that the $\boldsymbol{r}^{*}\left(t_{i}, p_{s}\right)$ satisfies KKT conditions (i.e., Formula (6)).

$$
\text { Proof: }
$$

At the beginning, the algorithm executes the CO$\operatorname{STEP}\left(\Pi, D\left(t_{i}\right)\right)$ and the output is denoted $\boldsymbol{r}_{\Pi}^{(*)}$. Since $\boldsymbol{r}_{\Pi}^{(*)}$ is derived from Definition 1 and Theorem $1, r_{\Pi}^{(*)}$ must satisfy Equation (12) and $\theta \sum_{i=1}^{R} \frac{l_{i}}{r_{i}}=D$, then if we let $\mu_{k}=0$ for any $k$, there must exist an assignment such that all the conditions in Formula (6) hold except for $r_{k}^{(*)} \leq a_{k}$. Accordingly, $\boldsymbol{r}^{*}=\boldsymbol{r}_{\Pi}^{(*)}$ as long as $r_{k}^{(*)} \leq a_{k}$ for all $r_{k}^{(*)} \mathrm{s}$ in $\boldsymbol{r}_{\Pi}^{(*)}$.

If $r_{\Pi}^{(*)}$ cannot satisfy all the $R$ inequalities $\left(r_{k}^{*} \leq a_{k}\right.$, where $k=1,2, \cdots, R$ ), we need to further adjust the solution $r_{\Pi}^{(*)}$ to find the one completely satisfying the condition (6). In Algorithm 1, at this moment, all the $r_{k}^{(*)} \mathrm{s}$ such that $r_{k}^{(*)}>a_{k}$ will be selected and set to $a_{k}$. Without loss of generality, assuming there are $h_{1}$ such resource shares and they are denoted as $r_{1}, r_{2}, \cdots, r_{h_{1}}$. Obviously, each selected $r_{k}$ must satisfy $\mu_{k} \cdot\left(r_{k}-a_{k}\right)=0$ because $r_{k}=a_{k}$. On the other hand, Algorithm 1 will continue to execute $\operatorname{CO}-\operatorname{StEP}(\Gamma, C)$ on the rest $R-h_{1}$ dimensions, where $C=D\left(t_{i}\right)-\theta \sum_{k=1}^{h_{1}} \frac{l_{k}}{r_{k}}$. Likewise, all the $R-h_{1}$ new resource shares (each denoted by $r_{k}, k=h_{1}+1, \cdots, R$ ) must also satisfy $r_{h_{1}+1}: r_{h_{1}+2}: \cdots: r_{R}=\sqrt{\frac{l_{h_{1}+1}}{b_{h_{1}+1}}}: \sqrt{\frac{l_{h_{1}+2}}{b_{h_{1}+2}}}:$ $\cdots: \sqrt{\frac{l_{R}}{b_{R}}}$, and $\sum_{i=1}^{R} \frac{l_{i}}{r_{i}}=D$, thus if each of them meets the condition $r_{k} \leq a_{k}$, the $R-h_{1}$ new resource shares and the previously selected $h_{1}$ will together compose the solution satisfying the condition (6). If there are still $h_{2}\left(0<h_{2} \leq R-h_{1}\right)$ new resource shares violating $r_{k} \leq a_{k}$ in this round, Algorithm 1 will continue the adjustment until the $H$ th round such that either all the $R-\sum_{i=1}^{H} h_{i}$ remaining resource shares can satisfy $r_{k} \leq a_{k}$ or there are no remaining resource dimensions in $\Gamma$. In the former case, we can easily verify that all the $R$ resource shares satisfy the condition (6) simultaneously, composing an optimal solution; for the latter case, we could conclude that $\theta \sum_{i=1}^{R} \frac{l_{i}}{a_{i}} \geq D$, then there does not exist a feasible resource allocation to run the task within the specified deadline. In this situation, $\boldsymbol{r}^{*}=\boldsymbol{a}=\left(a_{1}, a_{2}, \cdots, a_{R}\right)^{T}$ will get the execution time closest to the deadline, and it will serve as the final solution.

Although Algorithm 1 is proved optimal for minimizing the payment cost within user-defined deadline for his/her task, the deadline still may not be guaranteed due to two factors, either bounded available resources or inaccurate workload vector information about the task. We propose the following lemma, which provides a necessary and sufficient condition of guaranteeing the task's deadline given accurate prediction and relatively sufficient resources. In next section, we will discuss how to guarantee task's deadline when performing the Algorithm 1 with even inaccurate workload vector.

Lemma 1: Given a task $t_{i}$ 's workload vector $\boldsymbol{l}\left(t_{i}\right)=\left(l_{1}\right.$, $\left.l_{2}, \cdots, l_{R}\right)^{T}$ and its deadline $D\left(t_{i}\right)$, and a candidate execution node $p_{s}$, then $t_{i}$ can be executed within $D\left(t_{i}\right)$ if and only if (i.e., $\Leftrightarrow$ ) Inequality (14) holds.

$$
\sum_{j=1}^{R} \frac{l_{j}\left(t_{i}\right)}{a_{j}\left(p_{s}\right)} \leq D\left(t_{i}\right)
$$

Proof:

To prove $\Leftarrow$ : If Inequality (14) holds, it is obvious there must exist a viable resource allocation $\boldsymbol{r}\left(t_{i}\right)\left(\preceq \boldsymbol{a}\left(p_{s}\right)\right)$, such that $\sum_{j=1}^{R} \frac{l_{j}\left(t_{i}\right)}{a_{j}\left(p_{s}\right)}=D\left(t_{i}\right)$. Hence, $t_{i}$ can be executed within $D\left(t_{i}\right)$.

To prove $\Rightarrow$ : If $t_{i}$ can be executed within $D\left(t_{i}\right)$, there must exist a viable resource allocation $r\left(t_{i}\right)$ such that $\sum_{j=1}^{R} \frac{l_{j}}{r_{j}} \leq D\left(t_{i}\right)$ and $\boldsymbol{r}\left(t_{i}\right) \preceq \boldsymbol{a}\left(p_{s}\right)$. Assuming Inequality (14) does not hold at the moment, i.e., $\sum_{j=1}^{R} \frac{l_{j}\left(t_{i}\right)}{a_{j}\left(p_{s}\right)}>$ $D\left(t_{i}\right)$, then, we could derive Inequality (15).

$$
\sum_{j=1}^{R} \frac{l_{j}\left(r_{i}\right)}{r_{j}\left(p_{s}\right)}<\sum_{j=1}^{R} \frac{l_{j}\left(t_{i}\right)}{a_{j}\left(p_{s}\right)}
$$

Accordingly, we can derive that there must exist a dimension for example $d_{k}$ such that $r_{k}\left(t_{i}\right) \geq a\left(p_{s}\right)$, which contradicts to the previous assumption that $r\left(t_{i}\right)$ is a viable solution $\left(\boldsymbol{r}\left(t_{i}\right) \preceq \boldsymbol{a}\left(p_{s}\right)\right)$.

\section{Optimality ANALYSis With InACCURATE INFORMATION}

In this section, we focus on such a question: what is the final upper bound of task execution length as compared to its predefined deadline $D$, when running it using the resource vector allocated under Algorithm 1 with inaccurately predicted workload information?

\subsection{Problem Description}

Although Algorithm 1's output is proved optimal, such a result relies on a strong condition, i.e., accurate task's workload vector. That is, each user needs to precisely 
predict the execution property (i.e., workload ratio) for his/her task, before constructing the resource allocation with minimized payment for its execution under a user-specified deadline. In some cases, the execution property could be easily estimated accurately. For instance, we can decide the workload ratio between the data to be read/written from/to disk and those to be downloaded/uploaded via network by comparing their data sizes. In many other cases, however, the execution property cannot be accurately estimated, such as computation-intensive applications whose execution times highly depends on the CPU-cycles to consume.

Definition 2: Suppose a task $t_{i}$ 's real workload vector is $l\left(t_{i}\right)$, while its workload vector used by our algorithm is $\boldsymbol{l}^{\prime}\left(t_{i}\right)$ subject to the Inequality (16), where $\alpha$ and $\beta$ are the lower bound and upper bound for the estimation ratio specified by user based on experiences or particular workload prediction methods such as [13], [14], [15].

$$
\alpha \leq \frac{l_{k}^{\prime}\left(t_{i}\right)}{l_{k}\left(t_{i}\right)} \leq \beta, k=1,2, \cdots R
$$

To illustrate the above definition, an example is given. Assuming the task $t_{i}$ 's real workload ratios range in $[0.125,1]$, and the workload vector $\boldsymbol{l}^{\prime}\left(t_{i}\right)$ used by Algorithm 1 will be set based on the task's historical execution records. Suppose each element $l_{k}^{\prime}\left(t_{i}\right)(k=1,2, \cdots R)$ will be set to 0.25 if the corresponding true workload fluctuates in $[0.125,0.5]$ and set to 0.75 if the true workload ranges within $(0.5,1]$. Then, we could get Inequality (17) below, where $\alpha=\frac{0.125}{0.25}=0.5$ and $\beta=\frac{0.5}{0.25}=2$.

$$
0.5 \leq \frac{l_{k}^{\prime}\left(t_{i}\right)}{l_{k}\left(t_{i}\right)} \leq 2, k=1,2, \cdots R
$$

Using the inaccurate prediction $\boldsymbol{l}^{\prime}\left(t_{i}\right)$ to perform the Algorithm 1, it is obvious that $t_{i}$ 's real execution time may surpass the expected execution deadline $D\left(t_{i}\right)$. Hence, one question is what the worst performance will get when using $\boldsymbol{l}^{\prime}\left(t_{i}\right)$ instead of $\boldsymbol{l}\left(t_{i}\right)$, compared to the expected deadline $D\left(t_{i}\right)$.

\subsection{Deadline Extension Ratio with Skewed Estima- tion of Execution Property}

For simplicity of description, we denote $r_{E}^{*}\left(=\left(r_{E 1}^{*}, r_{E 2}^{*}\right.\right.$, $\left.\left.\cdots, r_{E R}^{*}\right)^{T}\right)$ and $T_{E}^{*}\left(=\theta \sum_{k=1}^{R} \frac{l_{k}}{r_{E k}^{*}}\right)$ as the output of Algorithm 1 with the skewed workload prediction and the corresponding execution time, respectively ( $E$ here implies "Estimation with error"). Similarly, we denote $r_{I}^{*}\left(=\left(r_{I 1}^{*}, r_{I 2}^{*}, \cdots, r_{I R}^{*}\right)^{T}\right)$ and $T_{I}^{*}\left(=D=\theta \sum_{k=1}^{R} \frac{l_{k}}{r_{I k}^{*}}\right)$ as the output with real workload vector and the corresponding execution time, respectively ( $I$ here indicates "Ideal case $\left.^{\prime \prime}\right)$. Hence, our objective is to determine the upper bound of $\frac{T_{E}^{*}}{T_{I}^{*}}$, a.k.a., deadline extension ratio.

We partition the situation that Algorithm 1 would face to two categories, where $r_{E}^{(*)}$ refers to the optimal resource allocation with the constraint (4) (unlike the notation $\left.r_{E}^{*}\right)$ :

$$
\text { - } r_{E}^{*}\left(t_{i}\right)=r_{E}^{(*)}\left(t_{i}\right) \text {. }
$$

- $\boldsymbol{r}_{E}^{*}\left(t_{i}\right) \neq \boldsymbol{r}_{E}^{(*)}\left(t_{i}\right)$.

The first situation indicates that in terms of the skewed estimation of workload ratios, all the resource shares calculated by the initial CO-STEP in Algorithm 1 are always no greater than the corresponding capacities. That is, it is equal to the situation with the assumption that Inequality (18) holds.

$$
\boldsymbol{r}_{E}^{(*)}\left(t_{i}\right) \preceq \boldsymbol{a}\left(p_{s}\right)
$$

In contrast, the second one means that the initial COSTEP cannot fulfill the above condition, and the optimal allocation cannot be found unless a few more adjustment steps (line 5 8 of Algorithm 1).

As follows, we will first derive task $t_{i}$ 's execution time upper bound for the first category (i.e., Theorem 3), and then discuss the upper bound (i.e., Theorem 4) for the more generic case including the second category.

Theorem 3: Given a submitted task $t_{i}$ with a predefined deadline $D\left(t_{i}\right)$, a candidate execution node $p_{s}$ with unbounded resource capacity and a resource price vector (denoted $\boldsymbol{b}\left(p_{s}\right)$ ), and a skewed workload vector $\boldsymbol{l}^{\prime}\left(t_{i}\right)$ subject to Inequality (16), then the bound of execution time must satisfy Inequality (19), under the resource allocation $\boldsymbol{r}_{E}^{(*)}$.

$$
\begin{gathered}
\frac{1}{\beta} \cdot D\left(t_{i}\right) \leq T_{E}^{(*)}\left(t_{i}\right) \leq \frac{1}{\alpha} \cdot D\left(t_{i}\right) \\
\text { Proof: } \\
T_{E}^{(*)}=\theta \sum_{k=1}^{R} \frac{l_{k}}{r_{E k}^{(*)}}=\theta \sum_{k=1}^{R} \frac{l_{k}}{\left(\frac{\theta}{D} \sum_{i=1}^{R} \sqrt{l_{i}^{\prime} b_{i}}\right) \sqrt{\frac{l_{k}^{\prime}}{b_{k}}}} \\
=\left(\frac{D}{\sum_{i=1}^{R} \sqrt{l_{i}^{\prime} b_{i}}}\right) \sum_{k=1}^{R} \frac{l_{k} \sqrt{b_{k}}}{\sqrt{l_{k}^{\prime}}} \leq \frac{D}{\sqrt{\alpha}} \cdot \frac{1}{\sqrt{\alpha}} \cdot \frac{\sum_{k=1}^{R} \sqrt{l_{k} b_{k}}}{\sum_{i=1}^{R} \sqrt{l_{i} b_{i}}}=\frac{D}{\alpha}
\end{gathered}
$$

The key of the above proof is based on the Inequality (16). Similarly, According to Inequality (16), we can also derive $T_{E}^{(*)} \geq \frac{D}{\beta}$.

Accordingly, Inequality (19) holds. It is easy to see that Inequality (19)'s bound is tight. Considering such a case: $\forall k, l_{k}^{\prime}\left(t_{i}\right)=\alpha l_{k}\left(t_{i}\right)$, then $T_{E}^{(*)}$ will be equal to $\frac{D}{\alpha}$.

Theorem 4: Given a submitted task $t_{i}$ with a predefined deadline $D\left(t_{i}\right)$, a candidate execution node $p_{s}$ with a limited available resource vector $\left(a\left(p_{s}\right)\right)$ and price vector $\boldsymbol{b}\left(p_{s}\right)$, and a skewed workload vector $\boldsymbol{l}^{\prime}\left(t_{i}\right)$ subject to Inequality (16), if Inequality (14) holds, then under the resource allocation $r_{E}^{*}$, the bound of execution time must conform to Inequality (20).

$$
\frac{1}{\beta} \cdot D\left(t_{i}\right) \leq T_{E}^{*}\left(t_{i}\right) \leq \frac{1}{\alpha} \cdot D\left(t_{i}\right)
$$

Proof: Without loss of generality, we denote $\Omega$ to be the set of resource dimensions accumulated by Line 5 of Algorithm 1, and the corresponding dimensions' indexes are $1,2, \cdots,|\Omega|$. That is, $r_{1}^{*}=a_{1}, r_{2}^{*}=a_{2}, \cdots, r_{|\Omega|}^{*}=a_{|\Omega|}$, while $r_{|\Omega|+1}<a_{|\Omega|+1}, \cdots, r_{R}<a_{R}$. Hence, we can get the following equation.

$$
T_{E}^{*}=\theta\left(\sum_{i=1}^{|\Omega|} \frac{l_{i}}{a_{i}}+\sum_{i=|\Omega|+1}^{R} \frac{l_{i}}{r_{E i}^{*}}\right)
$$

We could further prove the Inequality (20) as follows. 


$$
\begin{aligned}
& T_{E}^{*}=\theta \sum_{i=1}^{|\Omega|} \frac{l_{i}}{a_{i}}+\theta \sum_{k=|\Omega|+1}^{R} \frac{l_{k}}{r_{E k}^{*}}
\end{aligned}
$$

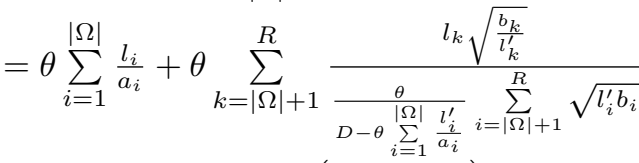

$$
\begin{aligned}
& =\theta \sum_{i=1}^{|\Omega|} \frac{l_{i}}{a_{i}}+\sum_{k=|\Omega|+1}^{R} \frac{\left(D-\theta \sum_{i=1}^{|\Omega|} \frac{l_{i}^{\prime}}{a_{i}}\right) \cdot l_{k} \sqrt{\frac{b_{k}}{l_{k}^{\prime}}}}{\sum_{i=|\Omega|+1}^{R} \sqrt{l_{i}^{\prime} b_{i}}} \\
& \leq \theta \sum_{i=1}^{|\Omega|} \frac{l_{i}}{a_{i}}+\left(D-\theta \sum_{i=1}^{|\Omega|} \frac{l_{i}^{\prime}}{a_{i}}\right) \sum_{k=|\Omega|+1}^{R} \frac{l_{k} \sqrt{\frac{b_{k}}{\alpha l_{k}}}}{\sum_{i=|\Omega|+1}^{R} \sqrt{\alpha l_{i} b_{i}}} \\
& =\theta \sum_{i=1}^{|\Omega|} \frac{l_{i}}{a_{i}}+\frac{1}{\alpha}\left(D-\theta \sum_{i=1}^{|\Omega|} \frac{l_{i}^{\prime}}{a_{i}}\right) \\
& \leq \frac{D}{\alpha}+\theta \sum_{i=1}^{|\Omega|} \frac{l_{i}}{a_{i}}-\frac{\theta}{\alpha} \sum_{i=1}^{|\Omega|} \frac{\alpha l_{i}}{a_{i}}=\frac{D}{\alpha}
\end{aligned}
$$

The key of the above proof is based on the Inequality (16). Similarly, According to Inequality (16), we can also derive $T_{E}^{*} \geq \frac{D}{\beta}$. Hence, Inequality (20) holds.

When $\Omega$ is empty, the lower bound \& upper bound of Inequality (20) can be reached as the upper bound \& lower bound of Inequality (16) are met respectively.

Remark: Let us review the Theorem 4 and discuss its significance. Inequality (20) implies that task $t_{i}{ }^{\prime} s$ execution time based on the optimal resource allocation of Algorithm 1 under inaccurate workload ratios has an upper bound, which is only determined by the lower bound of the inaccurate ratio $\alpha$. In principle, by leveraging this theoretical result, we can always provide the strict guarantee for user-preset deadline even with the wrong prediction of task's property, as long as there are relatively sufficient resources. In fact, what we need to do is just setting a stricter deadline $D^{\prime}$ according to the Formula (22) and preforming the Algorithm 1 based on $D^{\prime}$ instead of $D$. Then, the user task's deadline will be strictly limited under its expected value $D$ even though the workload ratio information is inaccurate (s.t. Inequality (16)). On the other hand, existing workload prediction work can be used to support how to determine the value of $\alpha$. For example, the polynomial regression method [16] can bound the prediction error in $10 \%$ (i.e., $\alpha=0.1$ ).

$$
D^{\prime}=\alpha \cdot D
$$

\section{Performance Evaluation}

\subsection{Experimental Setting}

We implement a web service based prototype that can compute a set of combined matrix-operations. Each matrix operation is called by some user task through a web service API and each task is executed in a VM container. Our algorithm is evaluated on such a real cluster environment. There are 10 physical nodes in the cluster, each owning 2 quad-core Xeon CPU E5540 (i.e., 8 processors per node) and $16 \mathrm{G}$ of memory. There are 60 VM-images (centos 5.2) kept by Network File System (NFS), so 60 VM-instances will be created at the bootstrap before our experiment. XEN 3.1 [17] serves as the hypervisor/VMM on each node and dynamically allocates various CPU speeds (or capabilities) to the VMinstances at run-time using credit scheduler.

Users can submit their computation request by editing their mathematical formulas. In our experiment, we make use of ParallelColt [18] to perform math computations, each consisting of a partially-ordered set of operations. ParallelColt [18] is such a library that can effectively calculate complex matrix-operations like matrix-matrix multiply, in parallel via multiple threads. Here is an example computation request, which is submitted as $\operatorname{Solve}\left(\left(A_{m \times n} \cdot A_{n \times m}\right)^{k}, B_{m \times m}\right)$. Such a computation task can be split into three steps (or subtasks) of different matrix-operations: (1) matrix-multiplication: $C_{m \times m}=A_{m \times n} \cdot A_{n \times m} ;$ (2) matrix-power: $D_{m \times m}=C_{m \times m}^{k}$; (3) Least squares solution of $D \cdot X=B$ based on QRDecomposition: Solve $\left(D_{m \times m}, B_{m \times m}\right)$. In our benchmark, we simulate a large number of user requests, each of which is composed of $3 \sim 15$ sub-tasks. Each subtask is constructed of three typical matrix-operations (i.e., matrix-multiply, matrix-power, and QR-matrixsolving(least-square)) with various parameters assigned. That is, each request contains many subtasks that are randomly selected from the above three types. We evaluate our algorithm under different competitive situation with different number (1 40) of tasks submitted simultaneously, thus there are 40 cases for each experiment which has 820 submitted tasks in total as observed.

In our system, each matrix-operation's workload is estimated based on the historical tracing records. The workload prediction formula is shown in Equation (23), where $j$ denotes the number of processors and $T\left(o p_{i}, j\right)$ indicates the execution time of running the matrix operation (denoted $o p_{i}$ ) on $j$ cores.

$$
l_{i}=\frac{1}{8} \sum_{j=1}^{8}\left(j \cdot T\left(o p_{i}, j\right)\right)
$$

Each user request (denoted as task $t_{i}$ ) is assigned with a deadline, which is a random value in $\left[\frac{1}{8} \cdot T_{1}\left(t_{i}\right), T_{1}\left(t_{i}\right)\right]$, where $T_{1}\left(t_{i}\right)$ means the estimated execution time when running the task $t_{i}$ on a particular core. Based on our experiment, the three matrix operations on one core will cost from 1 second to 1206 seconds as shown in Table 1, which implies a quite heterogenous nature. In Table $1, M, N, P$ refers to the matrix scale in the matrix-matrix-multiply and QR-Decomposition Solving, and $m$ indicates the value of exponent in the matrixpower computation. Users' prices of running the three individual matrix-operations are set to 1,2,3 respectively.

TABLE 1: Workload of Typical Matrix Operations (seconds/core)

\begin{tabular}{|l|l|l|l|l|l|l|l|l|l|l|}
\hline \multicolumn{4}{|c|}{ Matrix-Matrix-Multiply } & \multicolumn{3}{c|}{ QR-Decom. Solving } & \multicolumn{3}{c|}{ Matrix-Power } \\
\hline $\mathrm{M}$ & $\mathrm{N}$ & $\mathrm{P}$ & load & $\mathrm{M}$ & $\mathrm{N}$ & $\mathrm{P}$ & load & M & $\mathrm{m}$ & load \\
\hline 500 & 500 & 500 & 1.08 & 500 & 500 & 500 & 1.39 & 500 & 10 & 3.65 \\
\hline 1000 & 1000 & 1000 & 13.7 & 1000 & 1000 & 1000 & 6.1 & 500 & 20 & 4.2 \\
\hline 1500 & 1500 & 1000 & 31 & 1500 & 1500 & 1000 & 10.5 & 1000 & 10 & 55 \\
\hline 2000 & 1500 & 1000 & 40.2 & 2000 & 1500 & 1000 & 14.1 & 1000 & 20 & 67.2 \\
\hline 2000 & 2000 & 2000 & 118 & 2000 & 2000 & 2000 & 27.9 & 2000 & 10 & 457.7 \\
\hline 2500 & 2500 & 2500 & 242 & 2500 & 2500 & 2500 & 51 & 2500 & 20 & 1206 \\
\hline
\end{tabular}




\subsection{Experimental Results}

We first present the prediction effect over the historical records of the three matrix operations (as shown in Fig. 3), in that the approximation ratio of our optimal algorithm is based on the inaccuracy of the workload predicted, according to the analysis in Section 4. From this figure, we can clearly observe that the prediction method we used can make sure that the lower bound of the workload predicted (i.e., $\alpha$ 's value will be set close to 0.7 , where $\alpha$ is defined in Definition 2 and used in Theorem 3 and 4) is always lower than the real workload that is calculated after its execution.
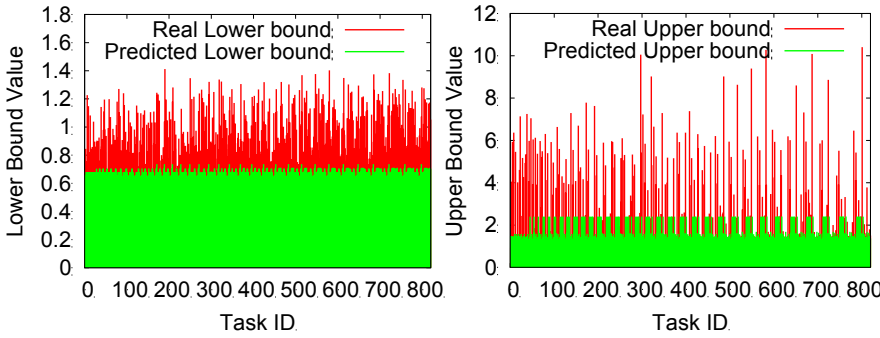

Fig. 3: Workload Prediction. (a) Lower Bound. (b) Upper Bound.

We evaluate our designed algorithm with and without the prediction-error-tolerant support. That is, the system will test the Algorithm 1 with the tuned stricter deadline $\left(D^{\prime}\right)$ or the original one $(D)$. We use Deadline Extension Ratio (DER) (defined as the ratio of task's final execution time to its deadline) to evaluate the statistical task execution lengths compared to their expected deadlines. We run 40 separate cases each with different number (1 40) of tasks, and show the lowest/average/highest level of DER for each case.

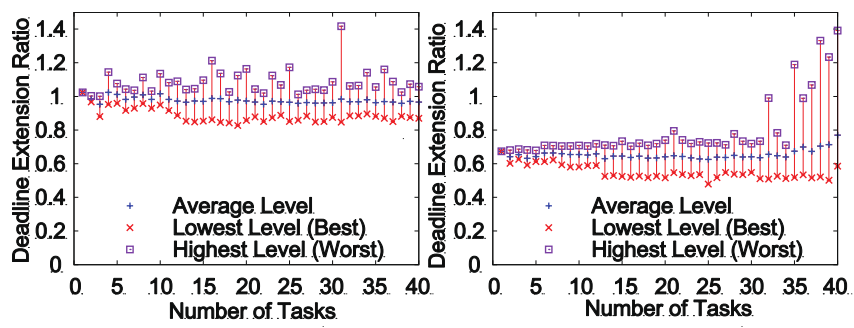

Fig. 4: Deadline Extension Ratio (DER). (a) $D^{\prime}=D$. (b) $D^{\prime}=\alpha \cdot D$.

We first show the experimental result by using the original deadline $D$ (i.e., $D^{\prime}=D$ ) in the algorithm. From Fig. 4 (a), we see that the tasks' execution times cannot be always guaranteed to be executed within their deadlines in the worst case, no matter how many tasks $(1 \sim 40)$ are submitted. Specifically, even though the system availability is relatively high (e.g., there are only several tasks submitted), the average value of deadline extension ratio is nearly to 1 and its highest value in the worst case is up to 1.2. This is mainly due to the inaccurate workload prediction with about $30 \%$ margin of errors as shown in Fig. 3. In comparison, Fig. 4 (b) shows the deadline extension ratio when the deadline $D^{\prime}$ is set to a stricter deadline $(\alpha \cdot D)$. When the number (denoted by $m$ ) of tasks submitted scales up to 30, all tasks' execution times can be kept nearly to about only 0.7 times as high as their preset deadlines $(D)$ at the worst situation (i.e., the highest level shown in the figure). With further increasing number of the submitted tasks, tasks' execution times cannot be always guaranteed because of the limited resource capacities (or the higher level of competitions on resources), but the mean level is still kept remarkably lower than 1 , which means that most of the tasks can still meet the QoS (i.e., large majority can be finished before deadlines). Note that there are only 10 physical machines in our experiment but much more than 10 tasks can be processed with guaranteed deadlines, which indicates a remarkably high level on service consolidation. This also implies a great potential in improving resource utilization by taking advantage of VM-multiplexing feature.

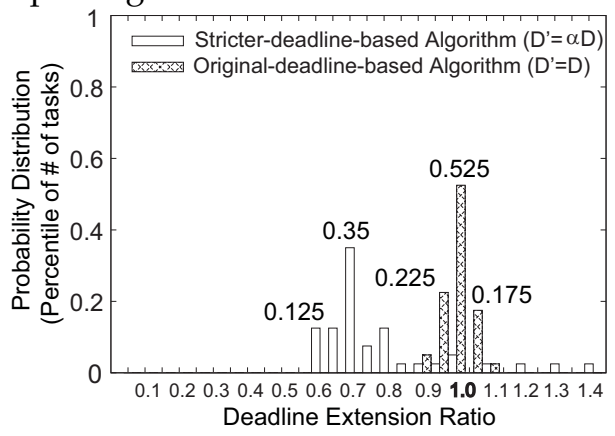

Fig. 5: Distribution of DER (the number of Tasks)

Fig. 5 presents the distribution of the deadline extension ratio (DER), in a competitive situation where there are 40tasks submitted. We observe that the stricterdeadline-based algorithm can more effectively limit the majority tasks' execution times to about 0.7 times as high as the user-specified deadlines (i.e., the original ones), but it may suffer from higher DER at the worst case. In comparison, the majority of tasks (about 52.5\%) under the original-deadline-based algorithm are completed within $0.95 \sim 1.0$ times of their deadlines, which still conforms to the deadline-guaranteed requirement; there are about $20 \%$ of tasks that would violate deadlines, most of which (17.5\%) are still finished within 1.05 times of deadlines.

Finally, we evaluate the fairness of task processing in the two cases, confirming the stability. Based on Jain's work [19], fairness index (higher value means higher fairness) is defined as Equation (24) whose value ranges in $[0,1]$, where $x_{i}$ refers to the DER of task $t_{i}$.

$$
F(\boldsymbol{x})=\frac{\left(\sum_{i=1}^{n} x_{i}\right)^{2}}{n \sum_{i=1}^{n} x_{i}^{2}}
$$

We present the experimental results about the fairness index of the DER in Fig. 6. As observed, the fairness index is always kept over 0.99 for both cases under the relatively uncompetitive situation (e.g., $m \leq 30$ ), and still kept about 0.95 in the case with higher competition (i.e., when $m>30$ ). Recall that there are only 10 physical machines used for resource provisioning in our experiment, which implies our solution's allocation effect is 


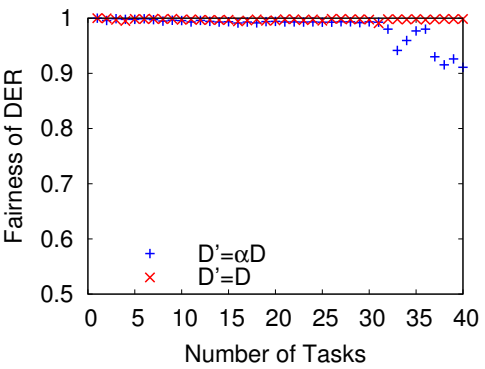

Fig. 6: Fairness Index of DER

confirmed to be quite stable to any task's execution under such a dense server consolidation. In addition, the main reason for the degradation of the fairness of DER in the competitive situation is that the tasks with higher priorities or the ones arriving earlier would be treated with higher service level in our experiment, which would definitely impact other lower-priority tasks' execution in the short-supply situation. In fact, guaranteeing the higher-priority tasks's QoS by sacrificing lower-priority tasks' benefit may also be considered a fairer treatment in many scenarios. Hence, for different applications, we can easily maximize the fairness level among all tasks by assigning the adaptive values for $\alpha$, which will be further studied in our future work.

\section{Related Work}

Traditional job scheduling [20] is often formulated as a kind of combinatorial optimization problem (or queuebased multi-processor scheduling problem [21], [22], [12]), due to the non-guaranteed performance isolation for multiple tasks running on the same machines. That is, most of the existing deadline-driven task scheduling solutions (from single cluster environment confined in LAN [23], [24] to the Grid computing environment suitable for WAN [25], [26]) are also strictly subject to the queueing model under which a single machine's multiple resources cannot be further split to smaller fractions at will. This will eventually cause the raw-grained resource allocation, relatively low resource utilization and sub-optimal task execution efficiency.

With the VM resource isolation technology being mature recently, it is viable to design more efficient resource allocation due to the fledged performance isolation among VMs running on the same machines. X. Meng et al. [27] proposed a VM multiplexing based resource allocation approach, which can successfully analyze the compatibility of any two different VMs (each with an application running atop it) on the same physical machines, and reschedule the combination of the VMs to improve the overall performance. However, it cannot guarantee high compatibility among more than two VMs on the same machine. Q-Clouds [28] is another wellknown system which can realize high consolidation of multiple VM-hosted applications, focusing on how to prevent inevitable performance interference among VMs from degrading user's QoS or enhancing corresponding users' payment unexpectedly.
Compared to the above existing works about VMmultiplexing resource allocation, our work aims to not only confine tasks' execution to be within their deadlines, but also minimize the payments for their users. This work will definitely benefit and motivate many Cloud users or service providers, who wish to minimize the infrastructure cost with the guaranteed QoS, actually already endeavored by many researchers. L. Wu et al. [29], for example, proposed a SLA-based resource allocation method, which is compatible to the heterogeneity of infrastructure and adaptable to the dynamic change of customer requests. It can maximize the profit of SaaS providers by minimizing the humber of SLA violations and the cost by reusing VMs. S. Chaisiri et al. [30] also aim to minimize the provisioning cost incurred to users by taking into account stochastic programming, robust optimization, and sample-average approximation together. M. Mao et al. [31], [32] present a Cloud autoscaling mechanism to automatically scale computing instances based on workload information and performance desire, also aiming to guarantee task's deadline with less payment. In comparison, our approach can be fundamentally proved optimal via the convex optimization theory, which we believe is a huge step forward especially from the perspective of theoretical analysis.

Most of the existing theoretical researches on Cloud computing [33], [34], [35] mainly focused on the relatively ideal scenarios by assuming tasks' workloads can be accurately predicted, simplifying the resource allocation problem. For example, J. Weinman [33] analyzed the penalty functions working in the workload aggregation and relative statistical effects, given a set of fixed task workloads to be used, while V. Petrucci et al. [34] proposed an optimization VM-based model to minimize the power and management cost by assuming that application's consumption can be predicted precisely by monitoring system. Unlike these works, we theoretically analyze the upper bound of task's execution time compared to its deadline and that of user-specified payment to the precise-prediction based result. By taking advantage of the derived bounds and approximation ratio, we can more effectively guarantee user tasks' QoS in terms of their demands. To the best of our knowledge, this is the first attempt to study how to minimize the payment cost in the cloud system, which can also tolerate the prediction errors of tasks' properties.

\section{Conclusion ANd Future Work}

In this paper, we propose a novel resource allocation algorithm for Cloud system that supports VMmultiplexing technology, aiming to minimize user's payment on his/her task and also endeavor to guarantee its execution deadline meanwhile. We can prove that the output of our algorithm is optimal based on the KKT condition, which means any other solutions would definitely cause larger payment cost. In addition, we analyze the approximation ratio for the expanded execution 
time generated by our algorithm to the user-expected deadline, under the possibly inaccurate task property prediction. When the resources provisioned are relatively sufficient, we can guarantee task's execution time always within its deadline even under the wrong prediction about task's workload characteristic. In the future, we plan to integrate our algorithms with stricter/orignal deadlines into some excellent management tools like OpenNebula, for maximizing the system-wide performance. Some queuing policies like earliest deadline first (EDF) will be studied to further reduce user payment especially in the short-supply situation. More complex scheduling constraints like the compatibility and security issue will also be taken into account.

\section{ACKNOWLEDGMENTS}

This research is supported by a Hong Kong RGC grant HKU 7179/09E and a Hong Kong UGC Special Equipment Grant (SEG HKU09).

\section{ReFERENCES}

[1] M. Armbrust, A. Fox, R. Griffith, A. D. Joseph, R. H. Katz, A. Konwinski, G. Lee, D. A. Patterson, A. Rabkin, I. Stoica, and M. Zaharia, "Above the clouds: A berkeley view of cloud computing," EECS Department, University of California, Berkeley, Tech. Rep. UCB/EECS-2009-28, Feb 2009.

[2] L. M. Vaquero, L. Rodero-Merino, J. Caceres, and M. Lindner, "A break in the clouds: towards a cloud definition," SIGCOMM Comput. Commun. Rev., vol. 39, no. 1, pp. 50-55, 2009.

[3] I. Foster and C. Kesselman, The Grid 2: Blueprint for a New Computing Infrastructure (The Morgan Kaufmann Series in Computer Architecture and Design). Morgan Kaufmann, November 2003.

[4] J. E. Smith and R. Nair, Virtual Machines: Versatile Platforms For Systems And Processes. Morgan Kaufmann, 2005.

[5] D. Gupta, L. Cherkasova, R. Gardner, and A. Vahdat, "Enforcing performance isolation across virtual machines in xen," in Proceedings of the ACM/IFIP/USENIX 2006 International Conference on Middleware (Middleware'06), New York, USA, 2006, pp. 342-362.

[6] J. N. Matthews, W. Hu, M. Hapuarachchi, T. Deshane, D. Dimatos, G. Hamilton, M. McCabe, and J. Owens, "Quantifying the performance isolation properties of virtualization systems," in Proceedings of the 2007 workshop on Experimental computer science (ExpCS '07). New York, USA: ACM, 2007.

[7] Amazon elastic compute cloud: on line at http://aws.amazon.com/ec2/.

[8] D. Milojicic, I. M. Llorente, and R. S. Montero, “Opennebula: A cloud management tool," Internet Computing, IEEE, vol. 15, no. 2, pp. 11 -14, march-april 2011.

[9] S. Boyd and L. Vandenberghe, Convex Optimization. Cambridge University Press, 2009.

[10] E. Imamagic, B. Radic, and D. Dobrenic, "An approach to grid scheduling by using condor-G matchmaking mechanism," in 28th International Conference on Information Technology Interfaces, 2006, pp. 625-632.

[11] B. Sharma, V. Chudnovsky, J. L. Hellerstein, R. Rifaat, and C. R. Das, "Modeling and synthesizing task placement constraints in google compute clusters," in Proceedings of the 2nd ACM Symposium on Cloud Computing (SOCC'11). ACM, 2011, pp. 3:1-3:14.

[12] H. Khazaei, J. V. Misic, and V. B. Misic, "Modelling of cloud computing centers using $\mathrm{m} / \mathrm{g} / \mathrm{m}$ queues," in ICDCS Workshops, 2011, pp. 87-92.

[13] Y. Wu, K. Hwang, Y. Yuan, and W. Zheng, "Adaptive workload prediction of grid performance in confidence windows," IEEE Transactions on Parallel and Distributed Systems, vol. 21, no. 7, pp. $925-938$, july 2010.

[14] S. Di, D. Kondo, W. Cirne,"Characterization and Comparison of Cloud versus Grid Workloads," in Proceedings of 14th International Conference on Cluster Computing (IEEE Cluster2012), 2012, pp. 230-238.
[15] Q. Zhang, , J. L. Hellerstein, and R. Boutaba, "Characterizing task usage shapes in google's compute clusters," in Large Scale Distributed Systems and Middleware Workshop (LADIS'11), 2011.

[16] L. Huang, J. Jia, B. Yu, B.G. Chun, P. Maniatis, and M. Naik, "Predicting Execution Time of Computer Programs Using Sparse Polynomial Regression," in 24th Conference on Neural Information Processing Systems (NIPS'10). 2010, pp. 1-9.

[17] P. Barham, B. Dragovic, K. Fraser, S. Hand, T. Harris, A. Ho, R. Neugebauer, I. Pratt, and A. Warfield, "Xen and the art of virtualization," in Proceedings of the nineteenth ACM symposium on Operating systems principles (SOSP'03). New York, NY, USA: ACM, 2003, pp. 164-177.

[18] P. Wendykier and J. G. Nagy, "Parallel colt: A high-performance java library for scientific computing and image processing," $A C M$ Trans. Math. Softw., vol. 37, pp. 31:1-31:22, September 2010.

[19] R. K. Jain, The Art of Computer Systems Performance Analysis: Techniques for Experimental Design, Measurement, Simulation and Modelling. John Wiley \& Sons, April 1991.

[20] C. Jiang, C. Wang, X. Liu, and Y. Zhao, "A survey of job scheduling in grids," in Proceedings of the joint 9th Asia-Pacific web and 8th international conference on web-age information management conference on Advances in data and web management (APWeb/WAIM'07). 2007, pp. 419-427.

[21] P. Crescenzi and V. Kann, A compendium of NP optimization problems. [Online]. Available: ftp://ftp.nada.kth.se/Theory/ViggoKann/compendium.pdf

[22] O. Sinnen, Task Scheduling for Parallel Systems (Wiley Series on Parallel and Distributed Computing). Wiley-Interscience, May 2007.

[23] K. Ramamritham, J. A. Stankovic, and W. Zhao, "Distributed scheduling of tasks with deadlines and resource requirements." IEEE Trans. Computers, vol. 38, no. 8, pp. 1110-1123, 1989.

[24] M. C. McElvany and P. D. Stotts, "Guaranteed task deadlines for fault-tolerant workloads with conditional branches," Real-Time Systems, vol. 3, no. 3, pp. 275-305, 1991.

[25] L. Zhao, Y. Ren, and K. Sakurai, "A resource minimizing scheduling algorithm with ensuring the deadline and reliability in heterogeneous systems," in 25th IEEE International Conference on Advanced Information Networking and Applications (AINA'11), 2011, pp. $275-282$

[26] W. Chen, A. Fekete, and Y. C. Lee, "Exploiting deadline flexibility in grid workflow rescheduling," in 11th IEEE/ACM International Conference on Grid Computing (Grid'10), 2010, pp. 105-112.

[27] X. Meng, C. Isci, J. Kephart, L. Zhang, E. Bouillet, and D. Pendarakis, "Efficient resource provisioning in compute clouds via vm multiplexing," in Proceeding of the 7th international conference on Autonomic computing (ICAC'10). ACM, 2010, pp. 11-20.

[28] R. Nathuji, A. Kansal, and A. Ghaffarkhah, "Q-clouds : Managing performance interference effects for qos-aware clouds," EuroSys2010, pp. 237-250, 2010.

[29] L. Wu, S. K. Garg, and R. Buyya, "Sla-based resource allocation for software as a service provider (saas) in cloud computing environments," in 11th IEEE/ACM International Symposium on Cluster, Cloud and Grid Computing (CCGRID'11), 2011, pp. 195-204.

[30] S. Chaisiri, R. Kaewpuang, B.-S. Lee, and D. Niyato, "Cost minimization for provisioning virtual servers in amazon elastic compute cloud," in 19th Annual IEEE/ACM International Symposium on Modeling, Analysis and Simulation of Computer and Telecommunication Systems (MASCOTS'11), 2011, pp. 85-95.

[31] M. Mao, J. Li, and M. Humphrey, "Cloud auto-scaling with deadline and budget constraints," in 11th IEEE/ACM International Conference on Grid Computing (Grid'10), 2010, pp. 41-48.

[32] M. Mao and M. Humphrey, "Auto-Scaling to Minimize Cost and Meet Application Deadlines in Cloud Workflows," in International Conference for High Performance Computing, Networking, Storage $\mathcal{E}$ Analysis (SC'11), 2011, pp. 49:1-49:12

[33] J. Weinman, "Smooth operator: The value of demand aggregation," 2011. [Online]. Available: http://joeweinman.com/Resources/Joe_Weinman_Smooth_Operator _Demand_Aggregation.pdf

[34] V. Petrucci, O. Loques, and D. Mossé, "A dynamic optimization model for power and performance management of virtualized clusters," in Proceedings of the 1st International Conference on EnergyEfficient Computing and Networking (e-Energy'10). New York, NY, USA: ACM, 2010, pp. 225-233.

[35] F. Chang, J. Ren, and R. Viswanathan, "Optimal resource allocation in clouds," IEEE International Conference on Cloud Computing, pp. 418-425, 2010. 


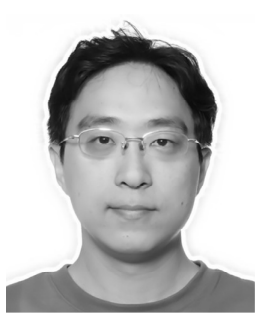

Sheng Di Sheng Di received his M.Phil degree from Huazhong University of Science and Technology in 2007 and Ph.D degree from The University of Hong Kong in 2011. He is currently a post-doctor researcher at INRIA. Dr. Di's research interest involves optimization of distributed resource allocation especially in P2P systems and large-scale Cloud computing platforms. His background is mainly on the fundamental theoretical analysis and practical system implementation. Contact him at the Department of Computer Science, The University of Hong Kong, Hong Kong, sdi@cs.hku.hk.

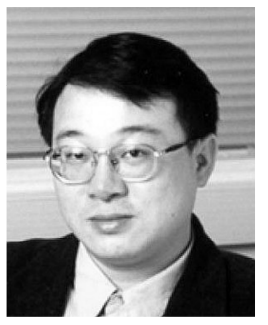

Cho-Li Wang Cho-Li Wang received his Ph.D. degree from University of Southern California in 1995. Dr. Wang's research interests include multicore computing, software systems for Cluster and Grid computing, and virtualization techniques for Cloud computing. He serves on the editorial boards of several international journals, including IEEE Transactions on Computers (2006-2010), Journal of Information Science and Engineering, and Multiagent and Grid Systems. $\mathrm{He}$ is the regional coordinator (Hong Kong) of IEEE Technical Committee on Scalable Computing (TCSC). Contact him at the Department of Computer Science, The University of Hong Kong, clwang@cs.hku.hk. 